\title{
Acute Effects of Mechanical Insufflation-Exsufflation on the Breathing Pattern in Stable Subjects With Duchenne Muscular Dystrophy
}

\author{
Ambra Cesareo MSc, Antonella LoMauro MSc, Marika Santi MSc MPT, Emilia Biffi PhD, \\ Maria G D'Angelo MD PhD, and Andrea Aliverti PhD
}

\begin{abstract}
BACKGROUND: Duchenne muscular dystrophy (DMD) is characterized by progressive degeneration, wasting, and weakness of skeletal musculature, including respiratory muscles. Cough is also compromised with disease progression. Among cough-augmentation techniques, mechanical insufflation-exsufflation (MI-E) has demonstrated several clinical benefits in patients with chronic airway secretion obstruction and muscular weakness. In clinical practice, the use of MI-E in DMD patients is also suggested when they are stable with no airway infections. However, there is a paucity of studies that consider the effect of MI-E specifically on stable DMD patients who have adapted to the use of MI-E. METHODS: Twenty subjects with DMD with no active upper airway or lung infections, who used MI-E device regularly at home, were enrolled. They received a single MI-E treatment consisting of 5 cycles of 5 insufflations-exsufflations with their customary settings. Volume variations during quiet breathing, vital capacity, and cough before and after treatment were measured with optoelectronic plethysmography (OEP). RESULTS: A decrease in breathing frequency $(P=.001)$ and the rapid shallow breathing index emerged $(P=.007)$, while cough peak flow (Spirometer $P=.86$, OEP $P=.58$ ), vital capacity (Spirometer $P=.78$, OEP total chest wall $P=.57$ ), and end-expiratory volumes (Total chest wall $P=.97$, Ribcage $P=.14$, Abdomen $P=.10$ ) were not affected by the treatment. An increment of the chest wall volume variation during the expiratory cough phase was identified $(P=$ $.001)$, particularly due to an increase in abdominal expansion $(P=.005)$. CONCLUSIONS: A single treatment of MI-E in subjects with stable DMD already adapted to the device can provide beneficial changes in breathing pattern through a significant decrease in breathing frequency and rapid shallow breathing. These findings suggest an improvement in short-term dyspnea, although there were no changes in lung-volume recruitment or unassisted cough peak flow. Key words: neuromuscular diseases; respiratory management; cough; thoraco-abdominal pattern; optoelectronic plethysmography; cough assist device. [Respir Care 2018;63(8):955-965. ( 2018 Daedalus Enterprises]
\end{abstract}

\section{Introduction}

Duchenne muscular dystrophy (DMD) is an X-linked recessive genetic disorder that is caused by mutations in

Ms Cesareo, Ms LoMauro, and Dr Aliverti are affiliated with the Dipartimento di Elettronica, Informazione e Bioingegneria, Politecnico di Milano, Milan, Italy. Ms Santi is affiliated with the Severe Acquired Brain Injury Unit, Scientific Institute IRCCS Eugenio Medea, Bosisio Parini, Lecco, Italy. Dr Biffi is affiliated with the Bioengineering Lab, Scientific Institute IRCCS Eugenio Medea, Bosisio Parini, Lecco, Italy. Dr D’Angelo is affiliated with the Neuromuscular Unit, Department of Neurorehabilitation, Scientific Institute IRCCS Eugenio Medea, Bosisio Parini, Lecco, Italy. the dystrophin gene, resulting in the absence of the dystrophin protein and a consequent rupture of the links between the cytoskeleton and the extracellular matrix. DMD is characterized by progressive degeneration, wasting, and weakness of skeletal musculature, including respiratory

\footnotetext{
Drs D'Angelo and Aliverti are co-last authors.

Ms Cesareo presented a version of this paper at the XIV Mediterranean Conference on Medical and Biological Engineering and Computing, held March 31 to April 2, 2016, in Paphos, Cyprus.
}

The authors disclose no conflicts of interest. 
muscles that ultimately become unable to ventilate properly. This leads to shallow breathing, hypoventilation, chest wall stiffness, sleep-disordered breathing, and respiratory failure. ${ }^{1-4}$

\section{See the Related Editorial on Page 1076}

With the progression of DMD cough, the fundamental defensive mechanism that guarantees airway clearance is also affected, resulting in the need for cough augmentation. ${ }^{5-8} \mathrm{~A}$ variety of cough-augmentation techniques, ranging from manual to mechanically assisted maneuvers, aim to improve the inspiratory and/or expiratory cough phase by maximally insufflating and/or deflating the lungs. ${ }^{9}$ Among these techniques, mechanical insufflation-exsufflation (MI-E), which alternatively insufflates and exsufflates the lungs to remove secretions, has demonstrated several clinical benefits in patients with chronic airway secretion obstruction and muscular weakness, including reduction of dyspnea and improvement of oxygen saturation, pulmonary morbidity, and hospitalization rates. ${ }^{6,10} \mathrm{In}$ a retrospective study, Stehling et al $^{11}$ studied the long-term effects of the regular use of MI-E in neuromuscular patients and demonstrated that it can improve vital capacity, which is the strongest predictor of mortality in neuromuscular patients. However, there is a paucity of studies that consider the effect of MI-E specifically in patients with DMD. Moreover, in clinical practice, the use of MI-E in patients with DMD is also suggested when they are stable (ie, having no airway infections). Under these circumstances, MI-E is used to mobilize the chest wall and to expand the lungs to avoid excessive stiffness and atelectasis. Nevertheless, further studies are needed to understand the acute effects of MI-E on the respiratory function of patients with DMD in stable state and already adapted to the use of a cough-assist device. In particular, there is little information about the capability of MI-E to acutely recruit lung volume.

The primary aim of this study was to evaluate the acute effects of a single treatment of MI-E in subjects with DMD who have no active upper airway or lung infection, in terms of lung recruitment changes, compliance of the respiratory system, breathing pattern, and volitional cough. For this purpose, optoelectronic plethysmography (OEP) is particularly suitable because it allows a detailed analysis of thoraco-abdominal kinematics and permits study of the

Correspondence: Ambra Cesareo MSc, Dipartimento di Elettronica, Informazione e Bioingegneria, Politecnico di Milano, 20133, Milan, Italy. E-mail: ambra.cesareo@polimi.it.

DOI: $10.4187 /$ respcare.05895

\section{QUICK LOOK}

\section{Current knowledge}

Mechanical insufflation-exsufflation (MI-E) is a coughaugmentation technique with several demonstrated clinical benefits in patients with chronic airway secretion obstruction and muscular weakness. In clinical practice, the use of MI-E in patients with Duchenne muscular dystrophy (DMD) is also suggested when they are stable and have no airway infections, but clinical studies have not examined the effects of MI-E use in this population in depth.

\section{What this paper contributes to our knowledge}

A single treatment of MI-E in stable subjects with DMD did not recruit lung volumes nor increase unassisted cough peak flow. Nevertheless, it was able to acutely decrease breathing frequency, thereby reducing rapid shallow breathing index.

action of different respiratory muscle groups. This technique is particularly useful in patients with DMD because it allows identification of variations in end-expiratory volumes, breathing pattern, vital capacity, and cough associated with the progression and disease severity; ${ }^{12-14} \mathrm{OEP}$ can be performed without requiring a mouthpiece or mask, which means it can be used during MI-E. ${ }^{15}$ Nevertheless, a specific validation of OEP use in patients with DMD is lacking in the literature, so we also compared the volume variations during vital capacity maneuvers and cough peak flow (CPF) as measured simultaneously with a portable spirometer and with OEP.

\section{Methods}

\section{Participants}

Diagnosis of DMD was based on clinical, genetic, or histological data. ${ }^{16}$ Among the patients with DMD who attended the Scientific Institute IRCCS Eugenio Medea for periodic clinical assessment, only those adapted to daily use of a cough-assistive device for at least $1 \mathrm{y}$ were included in this study.

For all subjects, clinical information including mutations in the DMD gene, use of noninvasive mechanical ventilation, years of use of cough-assistive devices, episodes of upper or lower airway infection in the last year, corticosteroids, cardiac function, severity of scoliosis, presence of spinal fusion, nutritional status, and use of percutaneous endoscopic gastrostomy were recorded. All subjects or parents signed a written informed consent form, as 


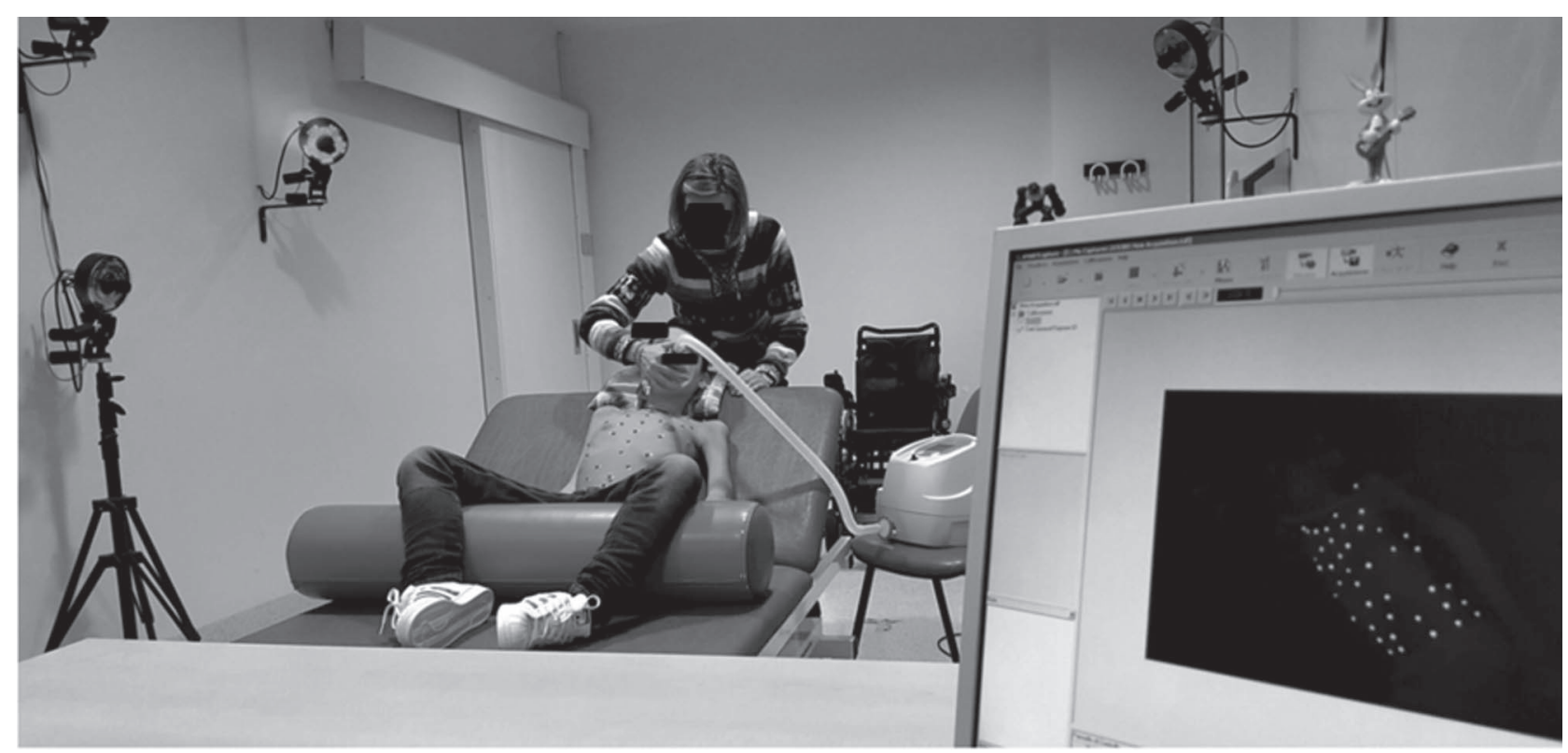

Fig. 1. Experimental set-up for the analysis of chest wall volumes with optoelectronic plethysmography. The subject is receiving a treatment with the cough-assistive device (mechanical insufflation-exsufflation) helped by an expert therapist. The optoelectronic cameras, the marker set, and the software used for the acquisition are also shown.

approved by the Local Ethical Committee of IRCCS Eugenio Medea, according to the Declaration of Helsinki. The study was performed at Scientific Institute IRCCS Eugenio Medea, Bosisio Parini, Lecco, Italy.

\section{Pulmonary Function Tests}

Data on Forced Vital Capacity (FVC), forced expiratory volume in 1 second $\left(\mathrm{FEV}_{1}\right)$, forced expiratory flow at 25$75 \%$ of the $\mathrm{FVC}$ maneuver $\left(\mathrm{FEF}_{25-75 \%}\right)$, forced expiratory flow at $50 \%$ of the FVC maneuver $\left(\mathrm{FEF}_{50 \%}\right)$, and peak expiratory flow (PEF) were acquired (Vmax series 22; SensorMedics, Yorba Linda, California). Moreover, subdivisions of lung volumes (functional residual capacity [FRC], residual volume [RV], and total lung capacity [TLC]) were obtained with the nitrogen washout technique.

\section{Measurements and Protocol}

Chest wall volume variations were acquired with OEP (OEP System; BTS, Milan, Italy) during all phases of the protocol. Slow vital capacity (SVC), performed with an inspiratory capacity followed by an expiratory vital capacity, and cough were acquired simultaneously using OEP and a portable spirometer (EasyOne Frontline, ndd Medical Technologies, Andover, Massachusetts). During the SVC maneuvers, vital capacity (VC) and inspiratory capacity (IC) were measured with a portable spirometer; during cough, the unassisted CPF was recorded. The spirometer was used with a face mask instead of a mouthpiece to avoid mouth leaks, because subjects with DMD may experience facial muscle weakness and may be unable to use a mouthpiece properly.

Each session took $\sim 1 \mathrm{~h}$, including the subjects' preparation phase and data acquisition. Subjects were asked to lie on a bed in a supine position, and the bed was adjusted to have the back support inclined at $\sim 45^{\circ}$ to reproduce the usual position for MI-E treatment. To allow a comfortable position during data acquisition, subjects' legs were supported by a pillow positioned under the knees (Fig. 1). The acquisition protocol was structured in 3 phases: pretreatment, treatment, and posttreatment (Fig. 2).

Pretreatment (T0) consisted of subjects being asked to perform an SVC maneuver (SVC T0) after a period of quiet breathing $(\mathrm{QB})$ to familiarize themselves with the laboratory setup, followed by 3 single maximal voluntary cough maneuvers (Cough T0) separated by at least $30 \mathrm{~s}$ of QB.

For the treatment phase, subjects received a treatment with the cough assist device after $60 \mathrm{~s}$ of QB (QB T0). Treatment consisted of 5 cycles of MI-E repeated 5 times (applications) spaced by at least $30 \mathrm{~s}$ of QB. Treatment was provided by an expert therapist who secured the mask to the subjects' face to minimize air leaks using subjects' personal devices, and the same settings used at home (eg, positive insufflation pressure, negative exsufflation pressure, durations, and inspiratory flow) were used during treatment.

During the posttreatment phase (T1), subjects were asked not to speak and to keep breathing quietly for at least $60 \mathrm{~s}$ (QB T1). At that time, 3 single maximal voluntary cough maneuvers (Cough T1) spaced by at least $30 \mathrm{~s}$ of QB and an SVC maneuver (SVC T1) were requested again. 


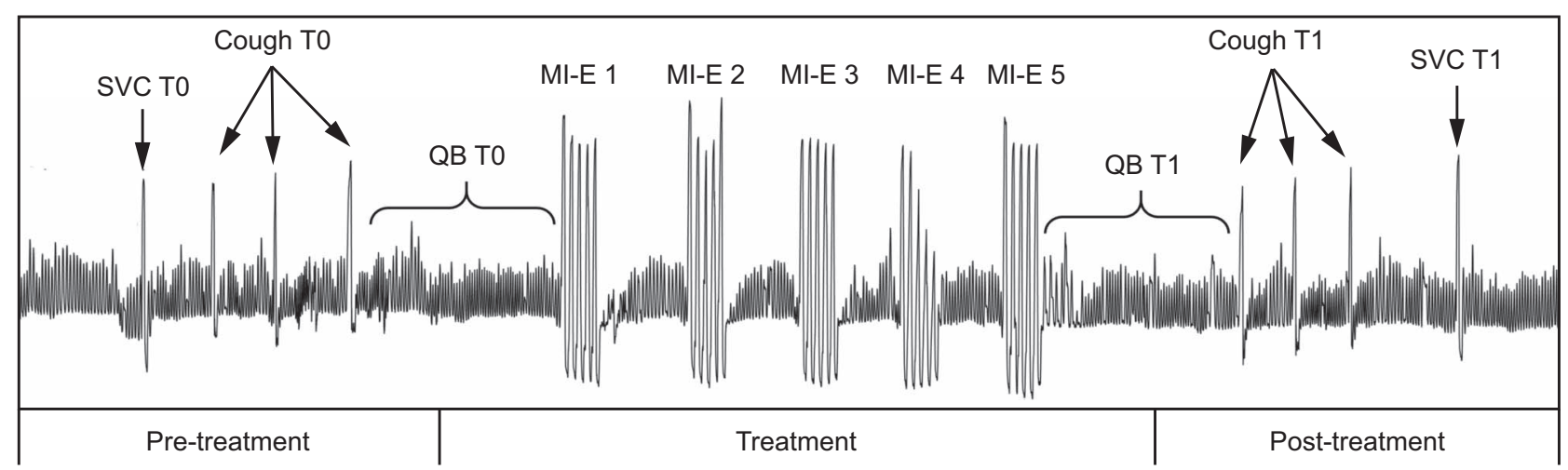

Fig. 2. Representation of the different phases of the acquisition protocol. Pretreatment, treatment, and posttreatment phases are shown; QB periods, SVC maneuvers, cough and MI-E cycles are highlighted. The tracing is an example of an optoelectronic plethysmography data (total chest wall volume variations) recorded from a participant. QB = quiet breathing, SVC = slow vital capacity, MI-E = mechanical insufflation-exsufflation.

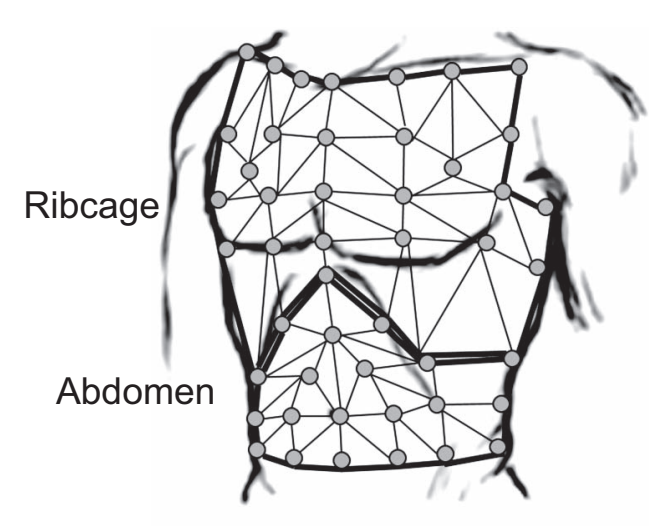

Fig. 3. Marker configuration for optoelectronic plethysmography acquisition. The schematic diagram shows the marker positioning on the front of the subject and the thoraco-abdominal surface triangulation defined to compute rib cage and abdominal volumes. The picture also shows the lateral markers positioned on the torso used to calculate the virtual plane defining the posterior part of the torso.

\section{Opto-Electronic Plethysmography (OEP) Parameters}

Total and compartmental chest wall volumes were measured with OEP. ${ }^{17-20}$ The system was composed of 8 infrared video cameras working at a sampling rate of $60 \mathrm{~Hz}$. It is able to compute the 3-dimensional coordinates of 52 retro-reflective markers placed, according to specific anatomical points, over the anterior chest wall surface of the subject lying on an adjustable bed. ${ }^{12,13,21}$ The posterior part of the trunk was defined by a virtual plane obtained by calculating a reference plane determined by the coordinates of the markers positioned laterally on the trunk (Fig. 3).21

From the 3-dimensional coordinates of the markers, specialized software provided continuous measurement of the volume enclosed by the chest wall surface $\left(\mathrm{V}_{\mathrm{CW}}\right)$ by applying Gauss's theorem. The chest wall was modeled with a bicompartmental model composed of the rib cage $\left(\mathrm{V}_{\mathrm{RC}}\right)$ and abdomen $\left(\mathrm{V}_{\mathrm{AB}}\right)$. The accuracy of the system during QB and the SVC maneuver has been previously tested by simultaneous measurements with a spirometer in healthy subjects in different positions. ${ }^{18,20}$

\section{Quiet Breathing}

Breathing and the thoraco-abdominal pattern during QB immediately before the first MI-E application (QB T0) and after the last MI-E application (QB T1) were analyzed, considering a normalized breath obtained by at least 4 breaths for both QB periods. The following breathing pattern parameters were computed on chest wall tracing: tidal volume $\left(\mathrm{V}_{\mathrm{T}}\right)$ calculated as the average of total $\mathrm{V}_{\mathrm{CW}}$ variations $\left(\Delta \mathrm{V}_{\mathrm{CW}}\right)$, total time of the respiratory cycle $\left(\mathrm{T}_{\mathrm{TOT}}\right)$, inspiratory and expiratory times $\left(\mathrm{T}_{\mathrm{I}}\right.$ and $\left.\mathrm{T}_{\mathrm{E}}\right)$, breathing frequency (f), minute ventilation $\left(\dot{\mathrm{V}}_{\mathrm{E}}=\mathrm{V}_{\mathrm{T}} \times \mathrm{f}\right)$, and rapid and shallow breathing index $\left(\mathrm{RSBI}=\mathrm{f} / \mathrm{V}_{\mathrm{T}}\right)$. To characterize the thoraco-abdominal pattern, the percentage contributions of the different compartments to $\mathrm{V}_{\mathrm{T}}\left(\% \Delta \mathrm{V}_{\mathrm{RC}}\right.$, and $\% \Delta \mathrm{V}_{\mathrm{AB}}$ ) were computed. To investigate changes in lung-volume recruitment, end-expiratory and end-inspiratory volumes of the chest wall and its compartments were also measured and reported as variations from the baseline volumes at end-expiration before treatment (QB T0). The assessment of end-expiratory and end-inspiratory volumes is important to understand how the respiratory muscles and ventilatory pattern adapt in response to the application of MI-E.

\section{Maneuvers}

SVC maneuvers and cough maneuvers were measured synchronously with OEP and a portable spirometer. For SVC analysis, the ribcage, abdominal, and total chest wall 


\section{Acute Effects of MI-E in Subjects With DMD}

volume changes during IC and VC were computed. For cough analysis, the instants of the start of cough inspiration, end of cough inspiration, and end of cough were identified. Ribcage, abdominal and total chest wall volume changes during the inspiratory cough phase and expiratory cough phase were respectively computed as the volume variation between start of cough inspiration and end of cough inspiration, and between end of cough inspiration and end of cough ${ }^{13,22,23}$ for each cough maneuver. The mean over the 3 cough maneuvers was then computed at $\mathrm{T} 0$ and $\mathrm{T} 1$ for each parameter.

To compute CPF, flow was obtained as the time derivative of the total chest wall volume $\left(\dot{V}=\mathrm{dV}_{\mathrm{CW}} / \mathrm{dt}\right)$ measured with OEP and filtered with a moving-average filter where each value was computed as the mean over 5 samples. At T0 and T1, expiratory peaks during the 3 cough maneuvers were selected and the CPF was computed as their mean.

\section{Statistical Analysis}

Mean values \pm SD were calculated for clinical, anthropometric, and pulmonary function parameters. Median values and interquartile ranges, expressed as the difference between 75th and 25th percentiles were computed for parameters related to $\mathrm{QB}, \mathrm{SVC}$, and cough. Results related to therse parameters are presented as Median [IQR].

To evaluate differences in QB, SVC, and cough parameters between pretreatment (T0) and post-treatment (T1), the parametric paired Student $t$ test was used. When data were not normally distributed, non-parametric Wilcoxon signed-rank test was used. We applied a Bonferroni correction for multiple dependent variables for $\mathrm{QB}$ analysis (breathing pattern, $n=6$; thoraco-abdominal pattern, $n=2$; end-inspiratory and end-expiratory volumes, $n=10$ ), for SVC analysis $(n=6)$, and for cough $(n=6)$. The significance level was set at $P<.05$.

Spearman's rank-order correlation was run to determine the relationship between measurements obtained with the portable spirometer and those obtained with OEP for VC and IC during SVC maneuvers and for CPF during cough. All of the data recorded during the SVC maneuvers at T0 and $\mathrm{T} 1$ were used to compute the correlations; all available data recorded for $\mathrm{CPF}$ during cough maneuvers at $\mathrm{T} 0$ and T1 were considered. To assess the agreement between OEP and portable spirometer measures, a Bland-Altman analysis was performed using VC and IC for the SVC maneuver and $\mathrm{CPF}$ for the cough maneuver, at $\mathrm{T} 0$ and $\mathrm{T} 1$. The mean percentage error was also computed for both maneuvers and expressed as mean $\pm \mathrm{SD}$. All computations were performed with SPSS version 23.0 for Windows (SPSS, Chicago, Illinois).
Table 1. Anthropometric and Clinical Characteristics of the Subjects

\begin{tabular}{lc}
\hline \hline Age, y & $20.80 \pm 4.50$ \\
Weight, $\mathrm{kg}$ & $53.90 \pm 16.31$ \\
Height, $\mathrm{cm}$ & $164.50 \pm 9.58$ \\
Body mass index, $\mathrm{kg} / \mathrm{m}^{2}$ & $19.67 \pm 4.98$ \\
Gene mutation, $n$ & \\
$\quad$ Deletion & 12 \\
$\quad$ Duplication & 3 \\
$\quad$ Point mutation & 5 \\
Scoliosis, $n$ & \\
$\quad$ Mild & 4 \\
$\quad$ Moderate & 5 \\
$\quad$ Severe & 5 \\
$\quad$ Spinal fusion & 6 \\
NIV, $n$ & 13 \\
Heart dysfunction, $n$ & 13 \\
Subjects with at least 1 respiratory tract & \\
$\quad$ infection in the last year, $n$ & \\
$\quad$ Upper airways & 12 \\
$\quad$ Lower airways & 5 \\
Duration of M-IE use, y & $4.3 \pm 1.69$ \\
& \\
\hline$N=20$ subjects. Data are presented as $n$ subjects or mean \pm SD. & \\
NIV $=$ noninvasive ventilation & \\
\hline
\end{tabular}

\section{Results}

\section{Clinical Data}

According to the inclusion criteria, 20 subjects with DMD who used an MI-E device were enrolled in the study. On average, they used the MI-E with a pressure level of $34 \pm 5 \mathrm{~cm} \mathrm{H}_{2} \mathrm{O}$ for both positive and negative pressures. Anthropometric and clinical characteristics of the subjects are shown in Table 1.

All of the subjects were wheelchair users, with an average loss of ambulation age of $10.4 \pm 2.5 \mathrm{y} ; 1$ subject at the time of the testing still had poor ambulation ability. In addition, 14 of 20 subjects had been treated with steroids for at least $2 \mathrm{y}$ in the past, 3 subjects were receiving steroid treatment at the time of evaluation, and 3 subjects had never used steroids.

All subjects presented with different severity levels of scoliosis, and 6 subjects underwent posterior spinal fusion on average $8.0 \pm 3.6$ y before this study. Subjects presenting with heart dysfunction (identified mainly with a left ventricle ejection fraction $<50 \%$ ) were using $\beta$ blockers or angiotensin-converting enzyme inhibitors or both treatments at the time of the study. Of the study group, 13 subjects were using noninvasive mechanical ventilation (NIV), and the average age at initiation of NIV was $19.5 \pm 3.3$ y. Eight subjects presented with good nutritional status (BMI $>18 \mathrm{~kg} / \mathrm{m}^{2}$ and BMI $\left.<25 \mathrm{~kg} / \mathrm{m}^{2}\right)$; 9 subjects presented with a pathological thinness together 
Table 2. Pulmonary Function

\begin{tabular}{|c|c|c|c|}
\hline \multicolumn{2}{|c|}{ Spirometry $(n=17)$} & \multicolumn{2}{|c|}{ Lung Volumes $(n=13)$} \\
\hline FVC, L & $0.97 \pm 0.55$ & TLC, L & $3.19 \pm 1.0$ \\
\hline FVC, $\%$ pred & $25.35 \pm 22.46$ & TLC, $\%$ pred & $56.33 \pm 25.74$ \\
\hline $\mathrm{FEV}_{1}, \mathrm{~L}$ & $0.83 \pm 0.46$ & $\mathrm{RV}, \mathrm{L}$ & $1.89 \pm 0.72$ \\
\hline $\mathrm{FEV}_{1}, \%$ pred & $29.65 \pm 39.39$ & $\mathrm{RV}, \%$ pred & $139.54 \pm 48.07$ \\
\hline $\mathrm{FEV}_{1} / \mathrm{FVC}, \%$ & $86.26 \pm 12.34$ & FRC, L & $2.14 \pm 0.68$ \\
\hline $\mathrm{FEF}_{25-75 \%}, \mathrm{~L} / \mathrm{s}$ & $1.12 \pm 0.79$ & FRC, $\%$ pred & $79.77 \pm 33.30$ \\
\hline $\begin{array}{c}\mathrm{FEF}_{25-75 \%}, \\
\% \text { pred }\end{array}$ & $23.35 \pm 16.75$ & & \\
\hline $\mathrm{FEF}_{50}, \mathrm{~L} / \mathrm{s}$ & $1.33 \pm 0.84$ & & \\
\hline $\mathrm{FEF}_{50}, \%$ pred & $29.35 \pm 22.83$ & & \\
\hline $\mathrm{PEF}, \mathrm{L} / \mathrm{s}$ & $2.14 \pm 1.03$ & & \\
\hline PEF, \% pred & $26.71 \pm 16.69$ & & \\
\hline \multicolumn{4}{|c|}{$\begin{array}{l}\text { Data are expressed as mean } \pm \text { SD. } \\
\mathrm{FEF}_{25-75 \%}=\text { forced expiratory flow during the middle half of the } \mathrm{FVC} \text { maneuver } \\
\mathrm{FEF}_{50}=\text { instantaneous flow at the moment the subject has exhaled } 50 \% \text { of } \mathrm{FVC} \\
\mathrm{PEF}=\text { peak expiratory flow } \\
\mathrm{TLC}=\text { total lung capacity } \\
\mathrm{FRC}=\text { functional residual capacity } \\
\mathrm{RV}=\text { residual volume }\end{array}$} \\
\hline
\end{tabular}

with swallowing disturbances (BMI $<18 \mathrm{~kg} / \mathrm{m}^{2}$ ); 3 subjects were overweight $\left(\mathrm{BMI}>25 \mathrm{~kg} / \mathrm{m}^{2}\right)$. Only 3 of $20 \mathrm{sub}-$ jects had a gastrostomy at the time of evaluation.

\section{Pulmonary Function}

Spirometric parameters $\left(\mathrm{FVC}, \mathrm{FEV}_{1}\right.$, Tiffeneau index, $\mathrm{FEF}_{25-75 \%}, \mathrm{FEF}_{50}$, and percentage with respect to the predicted values) were computed as mean \pm SD for 17 subjects. Lung volumes (TLC, RV, and FRC and percentage values) were computed as mean $\pm \mathrm{SD}$ and were available for 13 subjects. Three subjects did not perform the spirometry test (due to severe facial muscular weakness or macroglossia). The results of pulmonary function tests are reported in Table 2.

\section{Effects of MI-E Treatment}

\section{Quiet Breathing}

The breathing pattern parameters of all of the subjects at $\mathrm{T} 0$ and $\mathrm{T} 1$ are reported in Figure 4. Compared to T0, $\mathrm{V}_{\mathrm{T}}$ did not change and breathing frequency significantly decreased immediatly after the treatment $(P=.001)$. Therefore, the RSBI decreased significantly $(P=.007)$ immediatly after the treatment while minute ventilation decreased slightly, although not significantly. Expiratory time significantly increased $(P=.006)$ between T0 (Median [IQR] $1.43 \mathrm{~s}$ [0.83]) and $\mathrm{T} 1$ (1.89 s [0.76]), while inspiratory time did not change significantly (T0: $1.04 \mathrm{~s}$ [0.33] and T1: $1.21 \mathrm{~s}[0.30])$.
Thoraco-abdominal volumes were characterized by a higher $\% \Delta \mathrm{V}_{\mathrm{RC}}$ with respect to $\% \Delta \mathrm{V}_{\mathrm{AB}}$, which were similar between T0 $\left(\% \Delta \mathrm{V}_{\mathrm{RC}}\right.$ Median [IQR] 63.23 [35.52]; $\% \Delta \mathrm{V}_{\mathrm{AB}} 37.00$ [34.91]) and $\mathrm{T} 1\left(\% \Delta \mathrm{V}_{\mathrm{RC}} 61.74\right.$ [39.28]; $\% \Delta \mathrm{V}_{\mathrm{AB}} 38.28$ [39.13]). End-expiratory and end-inspiratory volumes did not significantly change after treatment (Table 3).

\section{Maneuvers}

Slow Vital Capacity. Considering all of the SVC maneuvers at $\mathrm{T} 0$ and $\mathrm{T} 1$, a strong, positive correlation emerged between measures obtained with the spirometer and those obtained with OEP, with statistically significant results ( $n=58, \mathrm{r}=0.93, P<.001)$; the equation of the regression line (Fig. 5A) is $\mathrm{y}=1.21 \mathrm{x}+0.07$. Bland-Altman analysis revealed a mean difference between OEP and spirometer data of $0.10 \pm 0.23 \mathrm{~L}$ and a mean percentage error of $13.96 \pm 28.85$. Figure 5 shows scatter plots (A and $\mathrm{B})$ and show Bland-Altman plots $(\mathrm{C}$ and $\mathrm{D})$ for $\mathrm{SVC}$ (A and C) and CPF (B and D) measures obtained with the spirometer and OEP.

Considering the data collected with the spirometer, no differences emerged between $\mathrm{T} 0$ and $\mathrm{T} 1$. In agreement with the spirometry data, no significant differences between $\mathrm{T} 0$ and $\mathrm{T} 1$ were found analyzing data collected with OEP for total chest wall VC and IC. Moreover, no significant differences emerged after the treatment in the compartmental contribution to total IC and VC compared to pretreatment conditions. Table 4 shows the data of $\mathrm{VC}$ and IC measured with the portable spirometer (Lung $\left[\Delta \mathrm{V}_{\mathrm{L}}\right]$ ) and with the OEP (total $\Delta \mathrm{V}_{\mathrm{CW}}[\mathrm{L}]$ and compartmental data $\left(\Delta \mathrm{V}_{\mathrm{RC}}[\mathrm{L}]\right.$ and $\left.\Delta \mathrm{V}_{\mathrm{AB}}[\mathrm{L}]\right)$.

Cough. Concerning CPF data, at T0 and T1, a positive significant correlation emerged between measures obtained with the spirometer and with OEP $(n=108, \mathrm{r}=0.76$, $P<.001$ ); the equation of the regression line (Fig. 5B) is $\mathrm{CPF}_{\mathrm{OEP}}=0.50 \times \mathrm{CPF}_{\text {spiro }}+59.67$. Bland-Altman analysis revealed a mean difference between OEP and spirometer data of $-41.66 \pm 58.41 \mathrm{~L} / \mathrm{min}$ and a mean percentage error of $-15.24 \pm 58.41$.

No differences emerged in CPF between T0 and T1 as measured with the portable spirometer (T0 Median [IQR] $163.00 \mathrm{~L} / \mathrm{min}$ [85.20]); T1 $164.60 \mathrm{~L} / \mathrm{min}$ [87.00]; $P=.86$ ) or OEP (T0 195.60 L/min [123.04]; T1 185.35 [159.07]; $P=.58)$. On the contrary, a significant increase of $\Delta \mathrm{V}_{\mathrm{CW}}$ volume emerged for the expiratory cough phase $(P=.001)$, particularly due to an increase in abdominal expansion $\left(\Delta \mathrm{V}_{\mathrm{AB}} \mathrm{T} 0\right.$ vs $\left.\mathrm{T} 1, P=.005\right)$. Total and compartmental chest wall volumes during cough maneuvers are shown in Table 5 . 

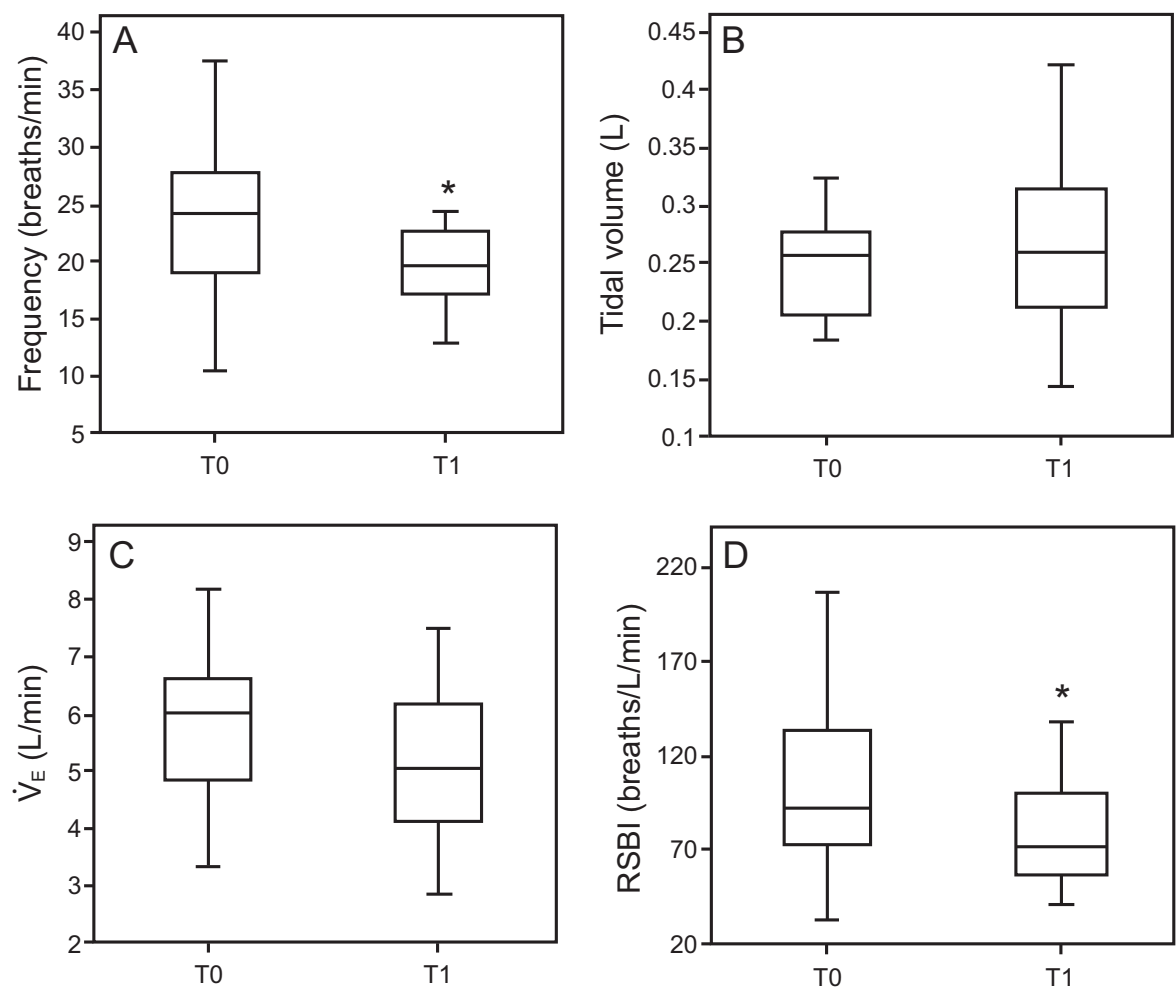

Fig. 4. Breathing pattern parameters. Box plots representing variables before (T0) and after (T1) MI-E treatment. Boxes show 25th to 75th percentile, and horizontal lines represent the median value. Whiskers show smallest and largest non-outliers. ${ }^{*} P=.001$ for breathing frequency; $P=.007$ for RSBI. RSBI = rapid and shallow breathing index.

Table 3. End-Expiratory and End-Inspiratory Volume Variations During Quiet Breathing

\begin{tabular}{lcrc}
\hline \hline & $\mathrm{T} 0$ & \multicolumn{1}{c}{$\mathrm{T} 1$} & $P$ \\
\hline$\Delta \mathrm{V}_{\text {eeRC }}, \mathrm{L}$ & $0(0)$ & $-0.02(0.09)$ & .14 \\
$\Delta \mathrm{V}_{\text {eeAB }}, \mathrm{L}$ & $0(0)$ & $0.01(0.05)$ & .10 \\
$\Delta \mathrm{V}_{\text {eeCW }}, \mathrm{L}$ & $0(0)$ & $0.00(0.08)$ & .97 \\
$\Delta \mathrm{V}_{\text {eiRC }}, \mathrm{L}$ & $0.13(0.09)$ & $0.17(0.18)$ & .74 \\
$\Delta \mathrm{V}_{\text {eiAB }}, \mathrm{L}$ & $0.08(0.06)$ & $0.09(0.06)$ & .55 \\
$\Delta \mathrm{V}_{\text {eiCW }}, \mathrm{L}$ & $0.25(0.06)$ & $0.28(0.13)$ & .65
\end{tabular}

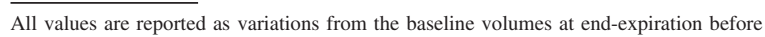
treatment (T0). Data are expressed as median (IQR), where the IQR is the difference between 75th and 25th percentiles.

$\Delta \mathrm{V}_{\mathrm{ee}}=$ change in end-expiratory volume

$\Delta \mathrm{V}_{\mathrm{ei}}=$ change in end-inspiratory volume

$\mathrm{RC}=$ ribcage

$\mathrm{AB}=$ abdomen

$\mathrm{CW}=$ chest wall

\section{Discussion}

This study examines the effects of a single treatment of MI-E on breathing and thoraco-abdominal patterns using OEP in subjects with DMD who have no active upper airway or lung infection, and evaluates QB, SVC, and cough. Similar to results reported by Winck et $\mathrm{al}^{10}$ and
Fauroux et al, ${ }^{7}$ MI-E did not affect thoraco-abdominal volume variations during QB. In this study, we have demonstrated that total and compartmental end-expiratory chest wall volumes did not change after MI-E treatment, which therefore cannot be considered equivalent to a recruitment maneuver. However, we recognized, immediately after the treatment, a change in breathing pattern characterized by a significantly lower breathing frequency and an improved RSBI that was significantly reduced. These findings are consistent with a recent study assessing the effects of volume recruitment-derecruitment maneuvers performed by means of MI-E on 9 subjects with DMD. ${ }^{15}$ The acute reduction of the breathing frequency after MI-E may have different origins because it is complexly regulated by the respiratory center in the medulla and pons of the brainstem through various inputs. These include signals from the peripheral chemoreceptors and central chemoreceptors, from the vagus nerve and glossopharyngeal nerve carrying input from stretch receptors and other mechanoreceptors in the lungs, bronchi, and bronchioles, as well as signals from the cerebral cortex and hypothalamus. The decrease in breathing frequency may be due to chemical-driven control of ventilation as a result of improved gas exchanges within the alveoli. This would be consistent with results obtained by Winck et al ${ }^{10}$ and Fauroux et al, ${ }^{7}$ who respec- 

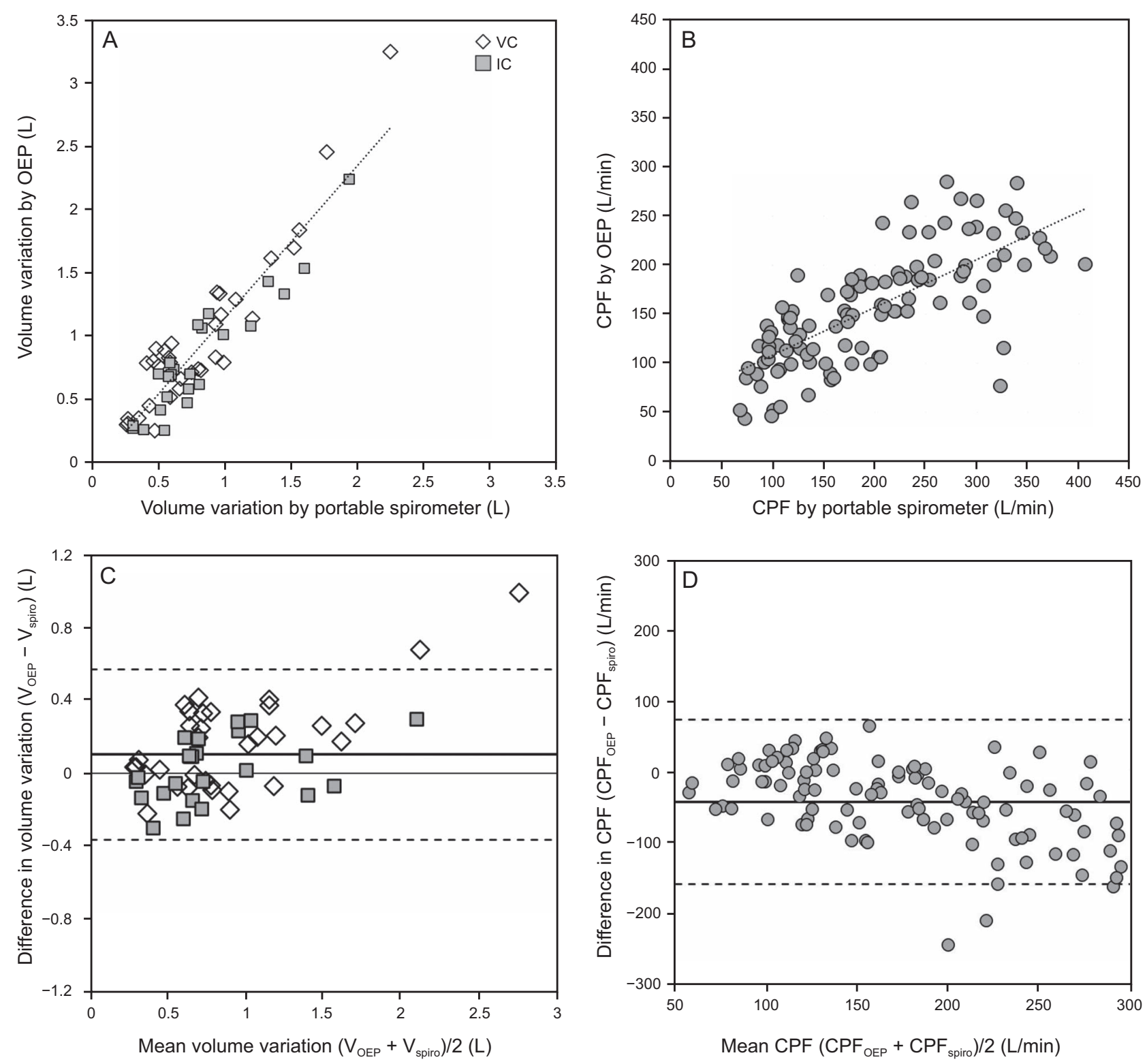

Fig. 5. Validation of OEP measurements during slow vital capacity $(A$ and $C)$ and $C P F(B$ and $D)$. (A) Vital capacity and inspiratory capacity measurements obtained by using OEP vs. portable spirometer at T0 and T1. Regression line of the data (vital capacity and inspiratory capacity together). (B) Scatter plot and regression line of CPF measures obtained by using OEP vs. portable spirometer at T0 and T1. (C) Bland-Altman plot of OEP vs. spirometer for vital capacity and inspiratory capacity measurements. (D) Bland-Altman plot OEP vs. spirometer for CPF measures. In C and D, the horizontal lines represent the mean of the differences (OEP-spirometer), while dotted lines show $t \pm 1.96 \mathrm{SD}$ of the differences (OEP-spirometer). OEP = optoelectronic plethysmography; CPF = cough peak flow; RSBI = rapid shallow breathing index.

tively identified an increase of $\mathrm{S}_{\mathrm{pO}_{2}}$ in subjects with chronic secretion encumbrance and a decrease of $\mathrm{P}_{\mathrm{ETCO}_{2}}$ in stable subjects after MI-E, and suggested that the improvements in alveolar ventilation were probably due to an increased recruitment of non-ventilated pulmonary zones and/or to the removal of mucous debris. Nevertheless, the immediate decrease in breathing frequency after the application of MI-E may also be an effect of insufflations on the modulation of ventilation control, driven by pulmonary stretch receptor activity. Increased stretch receptor activity shortens the duration of inspiration in all species, once a certain threshold volume is reached. Moreover, in some species, including dogs and rabbits, ${ }^{24-26}$ the expiratory discharge of slowly adapting stretch receptors determines expiratory duration and thus influences breathing frequency at rest. This has also been found in human neonates and children. ${ }^{27-30}$ This reflex is known as the Hering-Breuer inflation reflex. ${ }^{31}$ Adults exhibit a very weak Hering-Breuer 


\section{Acute Effects of MI-E in Subjects With DMD}

Table 4. Volume Variations During Slow Vital Capacity Maneuver

\begin{tabular}{lcccccccc}
\hline \hline & \multicolumn{3}{c}{ Vital Capacity } & & \multicolumn{3}{c}{ Inspiratory Capacity } \\
\cline { 2 - 3 } & T0 & T1 & $P$ & & T0 & T1 & $P$ \\
\hline $\mathrm{n}_{\mathrm{L}}, \mathrm{L}$ & $0.75(0.48)$ & $0.59(0.49)$ & .78 & & $0.73(0.41)$ & $0.67(0.51)$ & .88 \\
$\Delta \mathrm{V}_{\mathrm{CW}}, \mathrm{L}$ & $0.86(0.62)$ & $0.90(0.60)$ & .57 & & $0.75(0.52)$ & $0.73(0.57)$ & .45 \\
$\Delta \mathrm{V}_{\mathrm{RC}}, \mathrm{L}$ & $0.78(0.56)$ & $0.78(0.71)$ & .92 & & $0.71(0.48)$ & $0.69(0.60)$ & .60 \\
$\Delta \mathrm{V}_{\mathrm{AB}}, \mathrm{L}$ & $0.16(0.21)$ & $0.16(0.30)$ & .51 & & $0.12(0.22)$ & $0.14(0.22)$ & .08
\end{tabular}

Lung volume was recorded with a portable spirometer; all other volumes were measured with optoelectronic plethysmography during vital capacity and inspiratory capacity maneuvers

before (T0) and after (T1) MI-E treatment. Data are expressed as median (IQR), where the

$\mathrm{IQR}$ is the difference between 75 th and 25 th percentiles.

$\Delta \mathrm{V}_{\mathrm{L}}=$ change in lung volume

$\Delta \mathrm{V}_{\mathrm{CW}}=$ change in chest wall volume

$\Delta \mathrm{V}_{\mathrm{RC}}=$ change in ribcage volume

$\Delta \mathrm{V}_{\mathrm{AB}}=$ change in abdominal volume

Table 5. Volume Variations During Cough Maneuver Chest-Wall $\left(\Delta \mathrm{V}_{\mathrm{CW}}\right)$, Ribcage $\left(\Delta \mathrm{V}_{\mathrm{RC}}\right)$ and Abdominal $\left(\Delta \mathrm{V}_{\mathrm{AB}}\right)$ Volume Variations During Inspiratory and Expiratory Cough Phases Recorded by OEP Before (T0) and After (T1) MI-E Treatment

\begin{tabular}{lccccc}
\hline \hline & \multicolumn{2}{c}{ Inspiratory Cough Phase } & & \multicolumn{2}{c}{ Expiratory Cough Phase } \\
\cline { 2 - 3 } \cline { 5 - 6 } & $\mathrm{T} 0$ & $\mathrm{~T} 1$ & & $\mathrm{~T} 0$ & $\mathrm{~T} 1$ \\
\hline$\Delta \mathrm{V}_{\mathrm{CW}}, \mathrm{L}$ & $0.77(0.47)$ & $0.79(0.56)$ & & $0.83(0.46)$ & $0.92(0.59)^{*}$ \\
$\Delta \mathrm{V}_{\mathrm{RC}}, \mathrm{L}$ & $0.67(0.56)$ & $0.63(0.67)$ & & $0.69(0.48)$ & $0.65(0.62)$ \\
$\Delta \mathrm{V}_{\mathrm{AB}}, \mathrm{L}$ & $0.19(0.28)$ & $0.20(0.31)$ & & $0.23(0.22)$ & $0.26(0.24)^{*}$
\end{tabular}

Volume variations were measured with optoelectronic plethysmography before (T0) and after (T1) MI-E treatment. Data are expressed as median (IQR), where the IQR is the difference between 75 th and 25 th percentiles.

$* P=.001 \mathrm{~T} 1$ vs. T0.

$\Delta \mathrm{V}_{\mathrm{CW}}=$ change in chest wall volume

$\Delta \mathrm{V}_{\mathrm{RC}}=$ change in ribcage volume

$\Delta \mathrm{V}_{\mathrm{AB}}=$ change in abdominal volume

inflation reflex during quiet sleep, and the reflex can only be evoked in conscious children and adults at lung volumes well above the tidal range, when the inflation volume exceeds a critical threshold of about $1 \mathrm{~L},{ }^{29,30,32}$ similar to the volumes measured in our experiments with MI-E (1.22 $\pm 0.42 \mathrm{~L}$ on average). It has already been shown that pulmonary inflation with positive intra-alveolar pressure tends to reduce breathing frequency, prolonging the expiratory phase. ${ }^{33-36}$ Our results show a reduction of the breathing frequency immediately after treatment with MI-E, mainly due to an increase in expiratory time, which therefore could be explained by the Hering-Breuer Inflation reflex. However, from the data collected in this study, it is not possible to clearly discern if the frequency reduction is due to improved gas exchange within the alveoli or caused by the Hering-Breuer reflex. Besides, since the reduction of the breathing frequency remains statistically significant $(P=.02)$ even at the end of the acquisition protocol $(6.23 \pm 1.81 \mathrm{~min}$ after MI-E treat- ment), it may be a result of a summation effect of the chemical- and mechanical-driven mechanisms of ventilatory control.

Pulmonary function parameters (VC and IC) measured during SVC maneuvers, using both OEP and a portable spirometer, did not change after MI-E treatment, confirming the findings of Fauroux et al. ${ }^{7}$

Results for cough maneuvers showed that chest wall volume variations during the expiratory cough phases increased after the MI-E treatment, due to an increased abdominal contribution. Nevertheless, CPF values did not change as indicated by both spirometry and OEP data, which is in contrast to previous studies..$^{7,10}$ However, our results are not entirely comparable to those obtained in previous research. For example, Winck et al ${ }^{10}$ studied a mixed sample of subjects with chronic secretions (subjects with ALS, COPD, and other neuromuscular disorders, including only 1 subject with DMD), thus a difference in airway patency before and after treatment should be considered. Our subjects were stable and without infection, therefore the airways were patent at all study time points, which presumably did not influence CPF. Faurox et $\mathrm{al}^{7}$ enrolled a heterogeneous group of stable subjects (heterogeneous group of children with 4 DMD, 4 spinal muscular atrophy, and 9 congenital myopathies), but they were, on average, considerably younger than our participants. In addition, no information about the level of adaptation of the subjects to the use of MI-E was provided in the previous studies, ${ }^{7,10}$ whereas only subjects adapted to daily use of a cough-assist device for at least $1 \mathrm{y}$ were included in our study.

Another original aspect of this study is the use of OEP to allow a noninvasive, comprehensive assessment of not only chest wall volume variations during QB but also all breathing-pattern parameters, end-expiratory volumes, thoraco-abdominal partitioning during $\mathrm{QB}, \mathrm{SVC}$, and cough, and $\mathrm{CPF}$ as a time-derivative of chest wall volume variations during cough. The validation of OEP measurements during SVC maneuvers, originally provided in this study, confirms the reliability of the OEP technique in neuromuscular patients, who are limited in holding a mouthpiece during spirometric measurements. The slight overestimation of volumes measured with OEP compared to the measurements obtained with a portable spirometer, as demonstrated by the Bland-Altman analysis, might be attributed to air leaks at the mask and/or gas compression and decompression within the mask. A good correlation emerged for $\mathrm{CPF}$, but the regression line equation and mean percentage of error showed an underestimation of this measurement with OEP, which could be caused by the volume-time derivative needed to obtain flow from OEP measurements, and/or by the smoothing applied to the flux signal to detect peaks of flow. 


\section{Acute Effects of MI-E in Subjects With DMD}

The use of OEP, which we consider a strength of our study, could also be seen as a limitation because it is a technique that is not yet widely used in clinical centers and therefore does not represents a standard tool for respiratory function evaluation. The lack of information about the duration of the effects of MI-E over long periods represents a limitation of this study. Further investigations are needed a to verify if the beneficial effects on breathing patterns achieved with MI-E treatment are maintained over long-term periods, and to understand the effects of MI-E in patients experiencing airway infections and are not adapted to the use of MI-E. Moreover, measurements of $\mathrm{S}_{\mathrm{pO}_{2}}$ and/or end-tidal $\mathrm{CO}_{2}$ levels, which could have provided more information about the acute effects of MI-E on gas exchange, were not performed.

Our study has important strengths. First, our cohort of subjects was highly homogeneous in terms of age, diagnosis, and disease stage, compared to those used in previous studies. ${ }^{7,10}$ Second, the study reports the first validation of OEP during SVC maneuvers in subjects with DMD. Finally, the study has clinical implications, in that the use of MI-E in patients with DMD and without airway infection and secretion encumbrance is supported, considering the beneficial effects found in stable DMD patients.

The experimental protocol adopted in our study, including OEP measurements, could also be used for the optimization and personalization of MI-E settings, particularly when MI-E is introduced, but also for patient follow-up.

In conclusion, a short, single treatment of MI-E applied to subjects with clinically stable DMD who were already adapted to the use of the device did not acutely recruit lung volume nor increase $\mathrm{CPF}$. The treatment, however, affects the breathing pattern by decreasing the frequency, and thus reduces rapid and shallow breathing. This suggests beneficial effects in terms of reduced shortness of breath, at least in the short term.

\section{REFERENCES}

1. Bushby K, Finkel R, Birnkrant DJ, Case LE, Clemens PR, Cripe L, et al. Diagnosis and management of Duchenne muscular dystrophy, part 1: diagnosis, and pharmacological and psychosocial management. Lancet Neurol 2010;9(1):77-93.

2. Gozal D, Thiriet P. Respiratory muscle training in neuromuscular disease: long-term effects on strength and load perception. Med Sci Sports Exerc 1999;31(11):1522-1527.

3. Howard RS, Wiles CM, Hirsch NP, Spencer GT. Respiratory involvement in primary muscle disorders: assessment and management. Q J Med 1993;86(3):175-189.

4. Kalra M, Amin RS. Pulmonary management of the patient with muscular dystrophy. Pediatr Ann 2005;34(7):539-545.

5. Bach JR, Saporito LR. Criteria for extubation and tracheostomy tube removal for patients with ventilatory failure: a different approach to weaning. Chest 1996;110(6):1566-1571.

6. Bach JR, Ishikawa Y, Kim H. Prevention of pulmonary morbidity for patients with Duchenne muscular dystrophy. Chest 1997;112(4): 1024-1028.
7. Fauroux B, Guillemot N, Aubertin G, Nathan N, Labit A, Clément A, et al. Physiologic benefits of mechanical insufflation-exsufflation in children with neuromuscular diseases. Chest 2008;133(1):161-168.

8. Birnkrant DJ, Panitch HB, Benditt JO, Boitano LJ, Carter ER, Cwik VA, et al. American College of Chest Physicians consensus statement on the respiratory and related management of patients with Duchenne muscular dystrophy undergoing anesthesia or sedation. Chest 2007;132(6):1977-1986.

9. Bach JR, Barrow SE, Goncalves M. A historical perspective on expiratory muscle aids and their impact on home care. Am J Phys Med Rehabil 2013;92(10):930-941.

10. Winck JC, Gonçalves MR, Lourenço C, Viana P, Almeida J, Bach JR. Effects of mechanical insufflation-exsufflation on respiratory parameters for patients with chronic airway secretion encumbrance. Chest 2004;126(3):774-780.

11. Stehling F, Bouikidis A, Schara U, Mellies U. Mechanical insufflation/exsufflation improves vital capacity in neuromuscular disorders. Chron Respir Dis 2015;12(1):31-35.

12. LoMauro A, D'Angelo MG, Romei M, Motta F, Colombo D, Comi $\mathrm{GP}$, et al. Abdominal volume contribution to tidal volume as an early indicator of respiratory impairment in Duchenne muscular dystrophy. Eur Respir J 2010;35(5):1118-1125.

13. LoMauro A, Romei M, D'Angelo MG, Aliverti A. Determinants of cough efficiency in Duchenne muscular dystrophy. Pediatr Pulmonol 2014;49(4):357-365.

14. Romei M, d'Angelo M, LoMauro A, Gandossini S, Bonato S, Brighina E, et al. Low abdominal contribution to breathing as daytime predictor of nocturnal desaturation in adolescents and young adults with Duchenne muscular dystrophy. Respir Med 2012;106(2):276-283.

15. Meric H, Falaize L, Pradon D, Lacombe M, Petitjean M, Orlikowski $\mathrm{D}$, et al. Short-term effect of volume recruitment-derecruitment manoeuvre on chest-wall motion in Duchenne muscular dystrophy. Chron Respir Dis 2017;14(2):110-116.

16. Bushby K, Lochmüller H, Lynn S, Straub V. Interventions for muscular dystrophy: molecular medicines entering the clinic. Lancet 2009;374(9704):1849-1856.

17. Ferrigno G, Carnevali P, Aliverti A, Molteni F, Beulcke G, Pedotti A. Three-dimensional optical analysis of chest wall motion. J Appl Physiol (1985) 1994;77(3):1224-1231.

18. Cala SJ, Kenyon CM, Ferrigno G, Carnevali P, Aliverti A, Pedotti A, et al. Chest wall and lung volume estimation by optical reflectance motion analysis. J Appl Physiol (1985) 1996;81(6):2680-2689.

19. Aliverti A, Dellaca R, Pelosi P, Chiumello D, Pedotti A, Gattinoni L. Optoelectronic plethysmography in intensive care patients. Am J Respir Crit Care Med 2000;161(5):1546-1552.

20. Aliverti A, Dellacà R, Pelosi P, Chiumello D, Gattinoni L, Pedotti A. Compartmental analysis of breathing in the supine and prone positions by optoelectronic plethysmography. Ann Biomed Eng 2001;29(1):60-70.

21. Romei M, Mauro AL, D'angelo M, Turconi A, Bresolin N, Pedotti A, et al. Effects of gender and posture on thoraco-abdominal kinematics during quiet breathing in healthy adults. Respir Physiol Neurobiol 2010;172(3):184-191.

22. Guyton A, Hall J. Textbook of Medical Physiology. Philadelphia: WB Saunders; 1991.

23. Shah MD, Shah SM. The applied physiology of cough. Indian J Pediatr 2001;68(Suppl 2):S3-S10.

24. Davies A, Dixon M, Callanan D, Huszczuk A, Widdicombe J, Wise J. Lung reflexes in rabbits during pulmonary stretch receptor block by sulphur dioxide. Respir Physiol 1978;34(1):83-101.

25. Fishman NH, Phillipson EA, Nadel JA. Effect of differential vagal cold blockade on breathing pattern in conscious dogs. J Appl Physiol 1973;34(6):754-758.

26. Trenchard D. Role of pulmonary stretch receptors during breathing in rabbits, cats and dogs. Respir Physiol 1977;29(2):231-246. 


\section{Acute Effects of MI-E in Subjects With DMD}

27. Rabbette PS, Costeloe KL, Stocks J. Persistence of the Hering-Breuer reflex beyond the neonatal period. J Appl Physiol 1991;71(2):474-480.

28. Martin RJ, Nearman HS, Katona PG, Klaus MH. The effect of a low continuous positive airway pressure on the reflex control of respiration in the preterm infant. J Pediatr 1977;90(6):976-981.

29. Coleridge H, Coleridge J. Reflexes evoked from tracheobronchial tree and lungs. Comprehens Physiol 2011; doi.org/10.1002/ cphy.cp030212.

30. Rabbette PS, Stocks J. Influence of volume dependency and timing of airway occlusions on the Hering-Breuer reflex in infants. J Appl Physiol 1998;85(6):2033-2039.

31. Hering E, Breuer J. Self-control of breathing by the vagus nerve. Sitzber Deut Akad Wiss Wien 1868;57:672-690.

32. Hamilton RD, Winning AJ, Horner RL, Guz A. The effect of lung inflation on breathing in man during wakefulness and sleep. Respir Physiol 1988;73(2):145-154.
33. Graves C, Glass L, Laporta D, Meloche R, Grassino A. Respiratory phase locking during mechanical ventilation in anesthetized human subjects. Am J Physiol Regul Integr Comp Physiol 1986;250(5): R902-R909.

34. Simon PM, Zurob AS, Wies WM, Leiter JC, Hubmayr RD, Jensen ML, et al. Entrainment of respiration in humans by periodic lung inflations: effect of state and CO2. Am J Respir Crit Care Med 1999;160(3):950-960.

35. Tryfon S, Kontakiotis T, Mavrofridis E, Patakas D. Hering-Breuer reflex in normal adults and in patients with chronic obstructive pulmonary disease and interstitial fibrosis. Respiration 2001;68(2):140144.

36. Haberthür C, Guttmann J. Short-term effects of positive end-expiratory pressure on breathing pattern: an interventional study in adult intensive care patients. Crit Care 2005;9(4):R407-R415.

This article is approved for Continuing Respiratory Care Education credit. For information and to obtain your CRCE

(free to AARC members) visit 